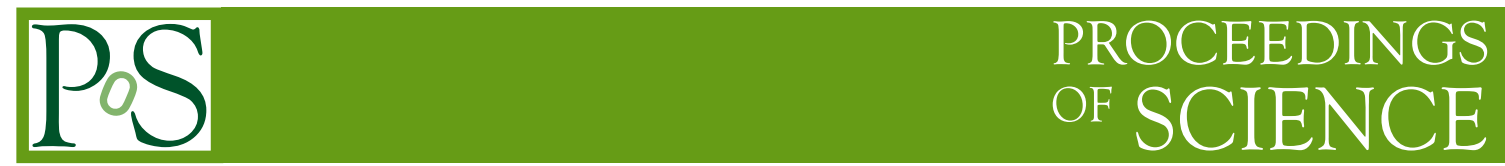

\title{
Simulation of lattice gauge action from the overlap operator
}

\author{
K.F. Liu* \\ Dept. of Physics and Astronomy, University of Kentucky, Lexington, KY 40506, USA \\ E-mail: liuepa.uky . edu
}

\begin{abstract}
We show the result of the lattice gauge field tensor which is derived from the classical continuum limit of the overlap Dirac operator. By analogous construction, it was recently proposed that the gauge action can be obtained from the overlap operator as well which is proportional to $\operatorname{Tr} a D_{o v}$. We discuss how to carry out Monte Carlo simulations of such a gauge action together with the overlap fermion.
\end{abstract}

XXIVth International Symposium on Lattice Field Theory

July 23-28, 2006

Tucson, Arizona, USA

${ }^{*}$ Speaker. 


\section{Introduction}

Lattice gauge actions are usually constructed from the link variable $U$. Similarly, the gauge field tensor is constructed from the clover-leaf like plaquettes.

The advent of Neuberger's overlap operator [1] has the implication in a new direction. The overlap Dirac operator is development to solve the fermion chirality problem in lattice QCD [2]. It does not have $O(a)$ error for the fermion operators. The small $O\left(\mathrm{~m}^{2} \mathrm{a}^{2}\right)$ errors and non-perturbative renormalization via current algebra relations and Ward identities make it a desirable fermion formulation for both light and heavy quarks [3]. An index theorem was formulated by Hasenfratz, Laliena, and Niedermayer [4] based on the operator at finite cut-off. It is then pointed out by Lüscher that the anomalous behavior of the fermion partition function under a flavor-singlet transformation is expressed by the index of the the overlap Dirac operator arising from the Jacobian, providing a clear understanding of the exact index theorem $[5,4]$ in the path-integral formalism. Following developments have seen the explicit derivation of the local lattice topological charge in terms of the local overlap operator via weak coupling expansion by Kikukawa and Yamada [6], explicit calculations without gauge coupling expansion by Adams [7], Fujikawa [8] and Suzuki [9], i.e.

$$
q_{L}(x)=\operatorname{tr}_{c s} \gamma_{5}\left(1-1 / 2 a D_{o v}(x, x)\right)
$$

where $D_{o v}$ is the overlap operator and the trace is over the color and Dirac spin indices. The lattice topological charge operator $q_{L}(x)$ so defined approaches the topological charge density $q(x)$ at the continuum limit,

$$
q_{L}(x) \underset{a \rightarrow 0}{\longrightarrow} a^{4} q(x)+O\left(a^{6}\right) .
$$

This formulation of the topological charge operator has not only been used to check the AtiyaSinger index theorem at finite lattice cut-off [10], the topological susceptibility [11], but has also been adopted to study the local topological structure of the vacuum [12, 13, 14, 15, 16, 17, 18]. It is with this operator that the sub-dimensional coherent sign structures have been discovered in 4-D QCD [14, 15, 16, 17] and 2-D CP(N-1) model [19]. It is argued that the chiral filtering of the overlap fermion is responsible for optimally filtering out the ultraviolet fluctuation to allow the structures to be revealed [20]. Indeed, other conventional topological charge operator constructed from the gauge links were used, but could not decipher the curvilinear structure observed with the overlap operator [19]. This leads to the possibility that gauge field tensor and gauge action derived from the overlap operator might be good alternatives to those from the gauge links directly. Recently, I. Horváth [21] proposed a formulation of lattice QCD wherein all elements of the theory (gauge action, fermionic action, theta-term, and other operators) are constructed from a single object, namely the lattice Dirac operator $D_{o v}$. In this talk, I will present the results of the derivation of the gauge field tensor as the classical continuum limit from the overlap operator. The detailed derivation [22] and the numerical evaluation [23] are under preparation and will be posted on the arXiv soon. I will also discuss how to simulate such an action with the Hybrid Monte Carlo algorithm.

\section{Gauge Field Tensor}

To begin with, we note that a gauge covariant operator which is a functional of $U$ satisfies the 
condition

$$
O\left(g^{-1} U g\right)=g^{-1} O(U) g,
$$

where $g$ is the local gauge transformation. It is obvious that the local operator with the color trace $\operatorname{tr}_{c} O(U)(x, x)$ is gauge invariant

$$
\operatorname{tr}_{c} O\left(g^{-1} U g\right)(x, x)=\operatorname{tr}_{c} g^{-1}(x) O(U)(x, x) g(x)=\operatorname{tr}_{c} O(U)(x, x) .
$$

Since the overlap operator, being a Dirac fermion operator, is gauge covariant and is not ultralocal, it is expected that the classical continuum limit of the trace in both the color and spin indices $\operatorname{tr}_{c s} \Gamma D_{o v}(x, x)$ will be the lowest dimensional local gauge operator which reflects the Lorentz structure of the gamma matrix $\Gamma$. Thus, it is not surprising that $\operatorname{tr}_{c s} \gamma_{5} D_{o v}(x, x)$ gives the local topological charge density at the continuum limit. Therefore, one expects that [21]

$$
\operatorname{tr}_{\mathrm{cs}}\left(D_{o v}(x, x)-D_{o v}^{0}(x, x)\right) \underset{a \rightarrow 0}{\longrightarrow} a^{4} t r_{c} F_{\mu v}^{2}+O\left(a^{6}\right),
$$

where $D_{o v}^{0}$ is the non-interacting overlap operator with gauge link $U$ set to unity. This has been verified with constant field and with numerical evaluation [23].

In addition to gauge invariant operators, one can obtain gauge covariant operators as well. Since $D_{o v}(x, x)$ is gauge covariant and a Lorentz scalar, one expects that $\operatorname{tr}_{s} \sigma_{\mu v} D_{o v}(x, x)$ with the spin index traced over will result in a lowest dimensional gauge covariant operator with the $\mu v$ indices in the classical continuum limit which is just the gauge field tensor [21]. In other words,

$$
\operatorname{tr}_{\mathrm{s}} \sigma_{\mu v} D_{o v}(x, x) \underset{a \rightarrow 0}{\longrightarrow} a^{2} F_{\mu v}+O\left(a^{4}\right) .
$$

We should note that the possibility of obtaining the lattice gauge field tensor from the overlap operator by expanding in $a$ was first pointed out by Niedermayer [24]. Also, it was suggested by Gattringer [25] that the field tensor can be defined through the square of various lattice Dirac operators, i.e. $t r_{s} \gamma_{\mu} \gamma_{v} D^{2}(x, y)$ with a weighted sum over $y$.

We have derived the classical limit following Adams [7] and Suzuki's method [9]. While details of the derivation will be given elsewhere [22], we shall present the results here.

$$
\begin{aligned}
& \operatorname{tr}_{\mathrm{s}} \sigma_{\mu \nu} a D_{o v}(x, x) \underset{a \rightarrow 0}{\longrightarrow} \\
& \int_{-\pi}^{\pi} \frac{d^{4} k}{(2 \pi)^{4}} \frac{-2}{\left(Z^{\dagger} Z\right)^{3 / 2}}\left[\left(m+r \sum_{\lambda}\left(c_{\lambda}-1\right) c_{\mu} c_{v}+2 r c_{\mu} s_{v}^{2}\right] a^{2} F_{\mu v}(x)+O\left(a^{4}\right),\right.
\end{aligned}
$$

where $s_{\mu}=\sin k_{\mu}, c_{\mu}=\cos k_{\mu}$ and

$$
Z^{\dagger} Z=\sum_{v} s_{v}^{2}+\left[m+r \sum_{\mu}\left(c_{\mu}-1\right)\right]^{2} .
$$

For the case where $r=1, m=1.368$ (which corresponds to Wilson $\kappa=0.19$ in the kernel), the proportional constant is -0.08156 for the overlap fermion [22]. This has been numerically verified [23]. One can try to use this operator to calculate glue properties, such as the glue momentum and angular momentum fractions in the nucleon to see if they are better than the glue operators constructed from the link variables as is in the case of the local topological charge. 


\section{Monte Carlo Simulation}

Take the proportional constant for the classical continuum limit in Eq. (2.3) to be $c$ which has been evaluated [23] as a function of the negative mass parameter $\rho$ in $D_{W}$, the kernel of the overlap operator $D_{o v}$. Then the lattice gauge action can be written as

$$
S_{g}=\frac{1}{2 c g^{2}} \operatorname{Tr}\left(D_{o v}-D_{o v}^{0}\right),
$$

where $\operatorname{Tr}$ denotes the trace over all indices, e.g. color, spin, and space-time. For a given lattice, the trace over the free quark overlap operator, i.e. $\operatorname{Tr} D_{o v}^{0}$ is a constant which has no effect on the Markov process to obtain equilibrium gauge configurations, we shall drop it. Noting that $D_{o v}$ obeys $\gamma_{5}$ hermiticity and satisfies Ginsparg-Wilson relation, we have

$$
S_{g}=\frac{1}{2 c g^{2}} \operatorname{Tr} D_{o v}=\frac{1}{4 c g^{2}} \operatorname{Tr}\left(D_{o v}+D_{o v}^{\dagger}\right)=\frac{1}{4 c g^{2}} \operatorname{Tr} D_{o v}^{\dagger} D_{o v}
$$

Now the lattice QCD partition function is

$$
Z=\int \mathscr{D} U d \bar{\psi}_{f} d \psi_{f} e^{-S_{g}+\sum_{f=1}^{N_{f}} \bar{\psi}_{f}\left(D_{o v}\left(m_{f}\right)\right) \psi_{f}},
$$

where $D_{o v}\left(m_{f}\right)$ is the overlap operator for a quark with mass $m_{f}$

$$
D_{o v}\left(m_{f}\right)=\rho D_{o v}+m_{f}\left(1-\frac{1}{2} D_{o v}\right)
$$

where $\rho$ is the negative mass parameter in $D_{W}$. Since $e^{T r M}=e^{\operatorname{Tr} \ln e^{M}}=\operatorname{det} e^{M}$, the gauge part of the partition function in Eq. (3.3) can be written in terms of a fictitious fermion field $\psi_{g}$ so that

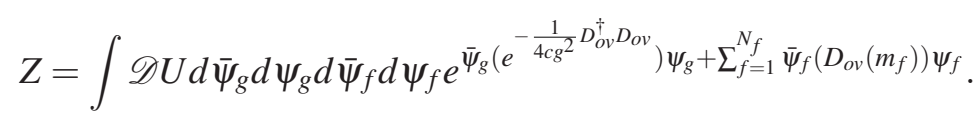

After integration of the fermion fields, it can be written as

$$
Z=\int \mathscr{D} U \operatorname{det}\left(e^{-\frac{1}{4 c g^{2}} D_{o v}^{\dagger} D_{o v}}\right) \prod_{f=1}^{N_{f}} \operatorname{det}\left(D_{o v}\left(m_{f}\right)\right) .
$$

We see that the gauge action plays the role of a UV-filtering for the fermion action (note that $c>0$ for the range of parameters for the Wilson kernel in the overlap operator with $r=1$ and $2>m>1$ [23]). The efficiency of an UV-filtered fermion determinant has been studied by Duncan, Eichten, and Thacker [26] and by Borici [27].

One way to carry out Monte Carlo simulation is to use pseudofermions to simulate the determinant. For example, one can equally split the gauge determinant and attach them to the fermion determinants of different flavor. In terms of the pseudofermions, it is

$$
Z=\int \mathscr{D} U d \phi_{f}^{*} d \phi_{f} e^{-\sum_{f=1}^{N_{f}} \phi_{f}^{*} e^{\frac{1}{4 c N_{f} g^{2}} D_{o v}^{\dagger} D_{o v}} D_{o v}^{-1}\left(m_{f}\right) \phi_{f}}
$$


We shall discuss two ways to approximate the pseudofermion action. Since $D_{o c}$ is normal, i.e. $\left[D_{o v}^{\dagger}, D_{o v}\right]=0$, one can write

$$
\phi_{f}^{*} e^{\frac{1}{4 c N_{f} g^{2}} D_{o v}^{\dagger} D_{o v}} D_{o v}^{-1}\left(m_{f}\right) \phi_{f}=\phi_{f}^{*} e^{\frac{1}{8 c N_{f} g^{2}} D_{o v}^{\dagger} D_{o v}} D_{o v}^{-1}\left(m_{f}\right) e^{\frac{1}{8 c N_{f} g^{2}} D_{o v}^{\dagger} D_{o v}} \phi_{f} .
$$

The range of eigenvalues of $D_{o v}^{\dagger} D_{o v}$ is from 0 to $4 \rho^{2}$. If $\frac{1}{2 c N_{f} g^{2}}$ is about unity or less, one can consider the Chebyshev polynomial approximation to degree $M$

$$
e^{\frac{1}{8 c N_{f} g^{2}} D_{o v}^{\dagger} D_{o v}} \sim \sum_{i=1}^{M} c_{i}\left(D_{o v}^{\dagger} D_{o v}\right)^{i}
$$

Alternatively, one can perform a Chebyshev rational polynomial approximation for the operator $e^{\frac{1}{4 c N_{f} g^{2}} D_{o v}^{\dagger} D_{o v}} D_{o v}^{-1}\left(m_{f}\right)$ to degree $N$ for the Rational Hybrid Monte Carlo algorithm (RHMC) [28]. For the case of $2+1$ flavors, the pseudofermion action for the 2 degenerate flavors can be approximated by

$$
\phi^{*} e^{\frac{1}{6 c g^{2}} D_{o v}^{\dagger} D_{o v}}\left(D_{o v}^{\dagger} D_{o v}\left(m_{f}\right)\right)^{-1} \phi \sim \phi^{*} \sum_{i=1}^{N} \frac{a_{i}}{D_{o v}^{\dagger} D_{o v}+b_{i}} \phi,
$$

and the single flavor one approximated by

$$
\phi^{*} e^{\frac{1}{12 c g^{2}} D_{o v}^{\dagger} D_{o v}}\left(D_{o v}^{\dagger} D_{o v}\left(m_{f}\right)\right)^{-1 / 2} \phi \sim \phi^{*} \sum_{i=1}^{N} \frac{c_{i}}{D_{o v}^{\dagger} D_{o v}+d_{i}} \phi .
$$

In this case, the forces in the equation of motion in HMC come from the effective pseudofermion actions in Eqs. (3.10) and (3.11) which represent the combined gauge and fermion forces.

If one uses a multi-mass algorithm for inversion and the coefficients $b_{i}$ and $d_{i}$ are not smaller than $m_{f}^{2}$, the overhead of incorporating the gauge action in RHMC is negligible compared to the ordinary $2+1$ flavor simulation in HMC with the same inverter.

One can of course accelerate the Rational Hybrid Monte Carlo algorithm (RHMC) by splitting the determinant into fractional flavors [29, 30] with more pseudofermion fields and improve the overall efficiency as shown by Clark and Kennedy [29].

In summary, we have discussed possible Monte Carlo simulations of the lattice gauge action from the trace of the overlap operator, i.e. $\operatorname{Tr} D_{o v}$ together with the overlap fermion action in the context of HMC. By virtue of the fact that the overlap operator is exponentially local, the gauge action so defined is expected to behave like a chirally smeared action. Furthermore, the integrand of the gauge part of the partition function, written in terms of a determinant, appears to be an UVfilter for the fermion determinant. We should note that, similar to the overlap fermion action, this gauge action is not reflection positive. Also presented is our derived result of the lattice gauge field tensor as the classical continuum limit of $\operatorname{tr}_{\mathrm{s}} \sigma_{\mu v} D_{o v}(x, x)$. This can be used to calculate glue matrix elements in the hadrons and possibly glueballs.

This work is partially supported by DOE grant DE-FG05-84ER40154. We wish to thank I. Horváth, A. Alexandur and A. Kennedy for stimulating discussions. The author also wish to thank the hospitality of the Institute of Physics, Academia Sinica, Taipei, Taiwan, National eScience Center, Edinburgh and DESY-Zeuthen, Germany where the talk is prepared and the proceedings is written up during the visit of these places. 


\section{References}

[1] H. Neuberger, Phys. Lett. B417, 141 (1998), [hep-lat/9707022]; ibid. B427, 353 (1998), [hep-lat/9801031].

[2] For a review, see, for example, H. Neuberger, Annu. Rev. Nucl. Part. Sci. 51, 23 (2001), [hep-lat/0101006].

[3] K.F. Liu and S.J. Dong, Int. J. Mod. Phys. A20, 7241 (2005), [hep-lat/0206002].

[4] P. Hasenfratz, V. Laliena, and F. Niedermayer, Phys. Lett. B427, 125 (1998), [hep-lat/9801021].

[5] M. Lüscher, Phys. Lett. B428, 342 (1998), [hep-lat/9802011].

[6] Y. Kikukawa and A. Yamada, Phys. Lett. B448, 265 (1999), [hep-lat/9806013].

[7] D. Adams, Annals Phys. 296, 131 (2002), [hep-lat/9812003].

[8] K. Fujikawa, Nucl. Phys. B546, 480 (1999), [ hep-th/9811235].

[9] H. Suzuki, Prog. Theor. Phys. 102, 141 (1999), [ hep-th/9812019]; T. Fujiwara, K. Nagao, and H. Suzuki, JHEP 0209, 025 (2002), [hep-lat/0208057].

[10] J.B. Zhang et al., Phys. Rev. D65, 074510 (2002), [hep-lat/0111060].

[11] L. Del Debbio, L. Giusti, C. Pica, Phys. Rev. Lett. 94, 032003 (2005), [hep-lat/0407052].

[12] I. Horváth, S.J. Dong, T. Draper, N. Isgur, F.X. Lee, K.F. Liu, J. McCune, H.B. Thacker, and J.B. Zhang, Phys. Rev. D66, 034501 (2002), [hep-lat/0201008].

[13] I. Horváth, S.J. Dong, T. Draper, F.X. Lee, K.F. Liu, H.B. Thacker, and J.B. Zhang, Phys. Rev. D67, 011501 (2003), [hep-lat/0203027].

[14] I. Horváth, S.J. Dong, T. Draper, F.X. Lee, K.F. Liu, N. Mathur, H.B. Thacker, and J.B. Zhang, Phys. Rev. D68, 114505 (2003), [hep-lat/0302009].

[15] I. Horváth, Nucl. Phys. B710, 464 (2005), [hep-lat/0410046].

[16] I. Horváth, A. Alexandru, J.B. Zhang, Y. Chen, S.J. Dong, T. Draper, F.X. Lee, K.F. Liu, N. Mathur, S. Tamhankar, and H.B. Thacker, Phys. Lett., B612 21 (2005), [hep-lat/0501025].

[17] I. Horváth, A. Alexandru, J.B. Zhang, Y. Chen, S.J. Dong, T. Draper, K.F. Liu, N. Mathur, S. Tamhankar, and H.B. Thacker, Phys. Lett. B617, 49 (2005), [hep-lat/0504005].

[18] A. Alexandru, I. Horváth, and J.B. Zhang, Phys. Rev. D72, 034506 (2005), [hep-lat/0506018].

[19] S. Ahmad, J. T. Lenaghan, and H. B. Thacker, Phys. Rev. D72, 114511 (2005), [hep-lat/0509066].

[20] I. Horváth, [hep-lat/0605008].

[21] I. Horváth, [hep-lat/0607031].

[22] K.F. Liu, A. Alexandru, and I. Horváth, 'Gauge Field Tensor from the Overlap Operator', under preparation.

[23] A. Alexandru, and I. Horváth, and K.F. Liu, 'Classical Limits of Certain Gauge Operators Based on the Overlap Dirac Kernel', under preparation.

[24] F. Niedermayer, Nucl. Phys. Proc. Suppl. 73, 105 (1999), [hep-lat/9810026].

[25] C. Gattringer, Phys. Rev. Lett. 88, 221601 (2002), [hep-lat/0202002].

[26] C. Duncan, E. Eichten, and H. Thacker, Phys. Rev. D59, 014505P (1999), [hep-lat/9806020]. 
[27] A. Borici, Phys. Rev. D67, 114501 (2003), [hep-lat/0205011].

[28] I. Horváth, A. Kennedy, and S. Sint, Nucl. Phys. Proc. Suppl. 73, 834 (1999), [hep-lat/9809092].

[29] M. Clark and A. Kennedy, [hep-lat/0409134] and [hep-lat/0608015].

[30] B. Joo, I. Horváth, and K.F. Liu, Phys. Rev. D67, 074505 (2003), [hep-lat/0112033]. 